Revista de Comunicación y Salud, 2019, Vol. 9, no 2, pp. 17-26

Editado por Cátedra de Comunicación y Salud

ISSN: 2173-1675

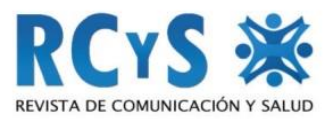

Enviado 11/09/2019

Aprobado 15/11/2019

\title{
APUNTES BIBLIOGRÁFICOS SOBRE SALUD SEXUAL EN MUJERES DE EDAD MEDIANA
}

\author{
Bibliographic notes on sexual health in middle-aged women
}

\begin{abstract}
Aime Napoles Betancourt ${ }^{1}$. Policlínico Federico Capdevila, La Habana, Cuba. aimenb@infomed.sld.cu
\end{abstract}

Ada Caridad Alfonso Rodríguez. Sociedad Cubana Multidisciplinaria para el Estudio de la Sexualualidad, La Habana, Cuba.

\section{Resumen}

En la mujer la línea de separación entre la adulta joven y la mujer de la tercera edad, es la mujer de mediana edad. El aumento de la esperanza de vida trae aparejado el incremento de mujeres que viven en este período del ciclo vital. La salud sexual requiere de un enfoque positivo y respetuoso de la sexualidad y las relaciones sexuales, Los estudios sobre el climaterio y la menopausia han reorientado la investigación en la última década, hacia aspectos cualitativos con la intención de profundizar la comprensión de las diferencias y a la vez demostrar con datos etnográficos que la experiencia de la mujer en esta etapa del ciclo vital, era captada de manera parcial por sus métodos de comprensión refieren los factores de riesgo que pudieran generar impacto negativo en la función sexual de la mujer en esta etapa de la vida. Todos los cambios biológicos y fisiológicos por los que transita la mujer en la edad mediana son parte de la evolución misma en la biología humana por lo que estos no justifican en sí mismo una perdida brusca y significativa de la actividad sexual.

Palabras clave: mujeres en la mediana edad, ciclo vital, menopausia, salud sexual.

\section{Abstract}

In women, the line of separation between the young adult and the elderly woman is the middle-aged woman. The increase in life expectancy brings the increase of women living in this period of the life cycle. Sexual health requires a positive and respectful

\footnotetext{
${ }^{1}$ Autor para correspondencia: Aime Napoles Betancourt aimenb@infomed.sld.cu
} 
approach to sexuality and sexual relations. Studies on climacteric and menopause have reoriented research in the last decade, towards qualitative aspects with the intention of deepening the understanding of differences and Once we have demonstrated with ethnographic data that the experience of women at this stage of the life cycle, was partially captured by their methods of understanding, they refer to the risk factors that could generate a negative impact on the sexual function of women at this stage of the lifetime. All the biological and physiological changes that women in middle age go through are part of the evolution itself in human biology, so they do not justify in themselves a sudden and significant loss of sexual activity.

Keywords: women in middle age, life cycle, menopause, sexual health.

\section{Cómo citar el artículo}

Napoles Betancourt, A. y Alfonso Rodríguez, A. C. (2019). Apuntes bibliográficos sobre salud sexual en mujeres de edad mediana. Revista de Comunicación y Salud, 9(2), 1726.

doi: http://doi.org/10.35669/revistadecomunicacionysalud.2019.9(2).17-26

\section{INTRODUCCIÓN}

La mujer de edad mediana, representa la línea de separación entre la adulta joven y la mujer de la tercera edad. Es la etapa de su ciclo vital, comprendida entre los 40 y los 59 años de edad, en la que acontece el climaterio, periodo donde se produce el tránsito de la etapa reproductiva a la no reproductiva (Sarduy Nápoles y Lugones Botell, 2006; FLASOG, 2016).

El concepto de Salud Sexual emitido por la Organización Mundial de la Salud (OMS), refiere que:

La Salud Sexual es un estado de bienestar físico, emocional, mental y social relacionado con la sexualidad y no solamente la ausencia de enfermedad, disfunción o incapacidad. La salud sexual requiere de un enfoque positivo y respetuoso de la sexualidad y las relaciones sexuales, así como de la posibilidad de tener experiencias sexuales placenteras y seguras, libres de coerción, discriminación y violencia (Así cambia el sexo según la edad, 2016; OMS, 2019).

Una aproximación a la salud sexual de las mujeres de edad mediana, remite a considerar el carácter pluridimensional y el conjunto de factores intervinientes, en cuanto a los componentes: biológico, motivo-afectivo-relacional y lo cognitivo; sin desdeñar el conjunto de condiciones, estructuras, fisiología, comportamientos y contextos socioculturales que permiten el ejercicio de la sexualidad. Incluye además, los sentimientos, la manera de expresar y de relacionarse, las representaciones, las subjetividades y los comportamientos de las mujeres de edad mediana (McCabe et al. 2016). 
El aumento de la esperanza de vida trae aparejado el incremento de mujeres que viven en este período del ciclo vital; la Organización Mundial de la Salud estima que para el año 2030 más de 1200 millones de mujeres, estarán por encima de los 40 años de edad, lo que supone que se habrá multiplicado por tres su número en apenas 40 años (Manzano Ovies, 2014; Navarro Despaigne \& Fontaine Semanat, 2001).

\section{SOBRE INVESTIGACIÓN EN SALUD SEXUAL}

En las últimas décadas, la salud sexual de las mujeres de edad mediana, y la sexualidad femenina han sido tratadas tanto en la literatura de Cuba, como en la internacional. La investigación en esta área, se ha enfocado en cómo viven las mujeres la sexualidad durante el climaterio, la posmenopausia y el envejecimiento; los resultados obtenidos expresan la existencia de una prevalencia de disfunción sexual de entre el 25\% y $43 \%$ en mujeres de mediana edad (Stalina Santisteban, 2011; Sarmiento Leiva, 2000).

Ya en la segunda mitad del siglo XX, comenzó la explosión sobre el conocimiento de la sexualidad; ello ha producido un cambio en la demanda de información y esta necesidad se ha incrementado siendo el tratamiento del tema, más abierto.

La primera investigación que relacionó las alteraciones de la esfera sexual con la fase menopáusica la realizó el Dr. Hallstrom, en 1977, y puso de manifiesto la existencia de una disminución del deseo sexual, de la capacidad para conseguir el orgasmo y de la frecuencia del coito en las mujeres (González Cárdenas, Bayarre Vera \& Hernández Melendez, 2019).

Los estudios sobre el climaterio y la menopausia han reorientado la investigación en la última década, hacia aspectos cualitativos con la intención de profundizar la comprensión de las diferencias y a la vez demostrar con datos etnográficos que la experiencia de la mujer en esta etapa del ciclo vital, era captada de manera parcial por sus métodos de comprensión (Couto Núñez \& Nápoles Méndez, 2014).

Una de las dimensiones del ser humano que históricamente ha creado diferencias en su interpretación y estudio, es la sexualidad, ya que existen diversas opiniones y enfoques respecto a su significado, a su importancia y a las relaciones con los otros aspectos de la naturaleza humana. De ahí, que cada cultura aborda en forma distinta esta expresión natural (Alfonso Rodríguez, 2010).

\subsection{La edad mediana y elementos fisiológicos de la función sexual}

La mujer durante la edad mediana transita por el climaterio, proceso del cual hasta una fecha relativamente reciente eran escasos los conocimientos fisiológicos al respecto en esta etapa ocurre un importante evento: la menopausia que es el cese permanente de la menstruación y representa el fin de la vida fértil de la mujer, su diagnóstico es retrospectivo y se hará después de un periodo de amenorrea mayor de 12 meses, según la OMS (Sarduy Nápoles y Lugones Botell, 2006; FLASOG, 2016).

Revista de Comunicación y Salud, 2019, Vol. 9, n 2, pp. 17-26 
La respuesta sexual femenina está controlada por el sistema nervioso central, interviniendo el sistema nervioso parasimpático en la actividad general y los tejidos eréctiles, mientras que el sistema nervioso simpático, controla el orgasmo, siendo producto de cambios anatómicos, hormonales, vasculares y neuronales que ocurren en el organismo, interviniendo diversos neurotransmisores.

La respuesta sexual consta de 4 fases:

1. Deseo (libido): deseo de tener actividad sexual, incluyendo pensamientos sexuales, imágenes y deseos.

2. Excitación: sensación subjetiva de placer sexual, acompañada de cambios fisiológicos, que incluyen vasocongestión genital y aumento de la frecuencia cardíaca, presión arterial y frecuencia respiratoria.

3. Orgasmo: pico del placer sexual y liberación de la tensión sexual, contracciones rítmicas de los músculos perineales y de los órganos reproductivos

4. Resolución: relajación muscular y sensación general de bienestar después de la actividad sexual.

Se realizará un paréntesis a cerca de la excitación sexual, pues este es un estado relacionado con algunos sentimientos específicos, vinculado con los genitales; en las mujeres tiene tres vías principales de expresión:

- Excitación central: caracterizada por activación mental que produce sueños

- Eróticos, ilusiones o fantasías sexuales voluntarias que pueden activar la excitación periférica física, genital y no genital.

- Excitación periférica no genital: se expresa por aumento de la secreción salival, sudoración, vasodilatación cutánea, erección del pezón, etc.

- Excitación genital: se expresa por congestión de la vulva vestibular y el clítoris, además de lubricación vaginal (Levin, 1992; FLASOG, 2016).

La respuesta endocrina tiene un fundamental papel en el establecimiento del adecuado umbral para la óptima respuesta a los estímulos sexuales. Los estrógenos conservan la salud vaginal, coadyuvan a la lubricación y previenen la dispareunia, entretanto los andrógenos modulan de forma directa a la fisiología de la vagina y del clítoris, al actuar en el tono muscular, tanto del tejido eréctil como de las paredes vaginales, facilitando la excitación y por ende la lubricación vaginal; asimismo influyen a nivel central, como activadores del deseo (Nelson, 2008).

El comportamiento sexual, aunque no debe modificarse, sufre algunos cambios ocasionados por la presencia de sequedad vaginal, la presencia de prolapsos, la aparición de las enfermedades crónicas dependientes de la ateroesclerosis, de la osteoporosis, de desequilibrio endocrino-metabólico y el cáncer entre otras. En esta etapa es frecuente observar una disminución de la libido, dispareunia y anorgasmia, en mujeres que hasta ese momento no tenían dificultades en el área sexual (Bajo Arenas, Lailla Vicens \& Xercavins Montosa, 2009; Navarro Despaigne, 2001). 
En cuanto a la función sexual es prudente recordar que los estrógenos conservan la salud vaginal, coadyuvan a la lubricación y previenen la dispareunia, entretanto los andrógenos, modulan de forma directa la fisiología de la vagina y del clítoris, al actuar en el tono muscular, tanto del tejido eréctil como de las paredes vaginales, facilitando la excitación y por ende la lubricación vaginal; asimismo influyen a nivel central, como activadores del deseo al igual que la disminución de los andrógenos en la merma del deseo sexual es decir que la respuesta endocrina tiene un fundamental papel en el establecimiento del adecuado umbral para la óptima respuesta a los estímulos sexuales (Davis \& Tran, 2001; Espitia De La Hoz \& Orozco-Gallego, 2018).

La mujer luego de la menopausia pasará un proceso secundario a las variaciones fisiológicas que presenta en el sistema hipotálamo-hipófisis-ovario, debido a la incapacidad del ovario para realizar la función folicular, no hay maduración folicular incrementándose los niveles de la hormona folículo estimulante (FSH) y le sigue la hormona luteinizante (LH); al no existir maduración folicular no existe síntesis y secreción de estradiol y de inhibina, y tampoco se produce progesterona, esta última es la que más disminuye, seguida de los estrógenos y los andrógenos, estos normalmente incrementan la sensación genital y estimulan la libido y el orgasmo, lo cual trae consigo cambios en los genitales externos pues aminoran las fibras de colágeno, el epitelio vaginal se adelgaza, se presenta un aumento en la fibrina y adelgazamiento del tejido conectivo con la respectiva pérdida de los pliegues, disminución de la elasticidad vaginal, sequedad vaginal, disminución de la respuesta vascular al estímulo sexual y cambios en el suelo pélvico, en particular el elevador del ano o diafragma pelviano y los músculos bulbocavernoso e isquiocavernoso, los cuales contribuyen cuando se contraen, con la penetración y las contracciones rítmicas del orgasmo, por ende sus cambios se expresan en dispareunia y disminución del deseo, también en demora y menor intensidad de la respuesta orgásmica (Navarro Despaigne, Méndez Gómez. \& Duany Navarro, 2017).

Dado que la función sexual y el desarrollo sexual humano abarca toda la vida, cada mujer tiene sentimientos, actitudes y creencias sexuales procesadas a través de una perspectiva individual, conformando las experiencias personales, acarreando consigo que los cambios varíen de una mujer a otra.

Los estudios de Navarro Despaigne et al. (2017) en Cuba, así como los de Espitia De La Hoz \& Orozco-Gallego (2018) en España, refieren los factores de riesgo que pudieran generar impacto negativo en la función sexual de la mujer en esta etapa de la vida, a continuación se hará referencia a una compilación de los mismos:

Factores Biológicos/orgánicos: Alteraciones hormonales, alteraciones urológicas o ginecológicas, enfermedades crónicas (Diabetes mellitus, dislipidemias, síndrome metabólico, hipertensión arterial, enfermedades reumatológicas y neurológicas, ...), uso de drogas legales (fármacos para el control de enfermedades indicadas por profesionales, alcohol y tabaco) e ilegales (marihuana, cocaína, opiáceos, anfetaminas, cannabis, ácido lisérgico). 
Factores psicológicos: Antecedentes de abuso físico o sexual, poca comunicación con la pareja, estrés, ansiedad, depresión, relaciones insatisfactorias, baja autoestima, conflictos con la imagen corporal.

Factores socioculturales: Educación sexual inadecuada, sobrecarga de género, conflictos con valores religiosos, personales o familiares, mitos y tabús sociales, desconocimiento de la propia anatomía y fisiología sexual, presencia de prejuicios sexuales, experiencia sexual traumática o incestuosa, inseguridad para el desempeño sexual, miedo a la intimidad sexual, expectativas irreales, trastornos sexuales personales anteriores, baja autoestima.

Factores dependiente de: pareja/vinculo: dificultades sexuales de la pareja, falta de pareja, falta de privacidad, técnica y habilidad de la pareja sexual, expectativas de una experiencia negativa, calidad de la relación y conflictos, mala comunicación, terceras partes, aburrimiento, decepción, resentimiento.

\section{ELEMENTOS RELACIONADOS CON LA AUTOPERCEPCIÓN COMO ELEMENTOS DE CIERRE DE LA PROPUESTA INVESTIGATIVA}

El cerebro adulto es a la vez plástico y resistente, y está siempre dispuesto a aprender. Las experiencias, los pensamientos, las acciones y las emociones cambian realmente la estructura del cerebro. La estructura cerebral no está predeterminada y fijada. Podemos alterar el desarrollo en marcha de nuestros cerebros y, por tanto, nuestras capacidades. Los seres humanos no somos prisioneros de nuestros genes o de nuestro entorno. Tenemos libre albedrío. Puede ser más duro para quienes tengan ciertos genes o entornos; pero ser «más duro» dista mucho de la predestinación. El cerebro está constantemente replanteando sus patrones conectivos en respuesta a todo lo que percibe, piensa o hace su poseedor. Cada experiencia, pensamiento, y emoción crea nuevas conexiones neuronales o refuerza las ya existentes (Casañas Velástegui, 2009).

En antropología del ciclo vital, la edad mediana está ubicada en la etapa de la adultez, por lo que la mujer llegará a la misma con un conocimiento y representación en su pensamiento que le permitirá tener una percepción de sí misma y su contexto.

La percepción conceptualmente ha tenido diferentes vertientes de estudio por las ciencias de la comunicación, la filosófica y la psicología, persistiendo como un proceso de formación de representaciones mentales con la función de realizar abstracciones a través de las cualidades, que definen lo esencial de la realidad externa, dicho también, que las percepciones, son los procesos mediante los cuales se registra todo con lo que se está interactuando y tiene significado, incluyen los valores, las tradiciones, los estereotipos, las vivencias y los conocimientos, que tienen los individuos sobre determinados aspectos de la vida (Oviedo Gilberto, 2004).

La autopercepción de salud, es la representación o idea que el individuo tiene acerca de su estado o condición presente de salud, expresada en términos valorativos de placer

Revista de Comunicación y Salud, 2019, Vol. 9, n 2, pp. 17-26 
o displacer, de satisfacción o insatisfacción y que puede o no corresponderse con el nivel de funcionamiento real de su organismo (Hernández Sánchez \& Forero Bulla, 2011).

La autopercepción de salud tanto del estado de salud general, como de la salud sexual, dependen de la historia de vida, estilo de vida, las condiciones de salud y trabajo tanto para las que laboran solo en casa, como para las que tienen doble jornada, la cultura y su construcción social, se individualiza en cada mujer, a través de su amplia dimensión de personalidad.

Los elementos que se mueven a raíz del declinar hormonal, estarán en relación con lo vivido en todas y cada una de las etapas de su vida, y los elementos socioculturales, la personalidad premórbida, la historia de vida y su construcción social, que conforman su personalidad, maneras de pensar y vivir y por ende las potencialidades y recursos psicológicos que le permitirán enfrentar el climaterio de disimiles maneras en cada mujer, así como la asimilación del envejecimiento, hacen que las mujeres de edad mediana constituyan un grupo altamente vulnerable en términos de salud ((Hernández Sánchez \& Forero Bulla, 2011; Orama Díaz, 2000; Artiles, 2007).

Todos los cambios biológicos y fisiológicos por los que transita la mujer en la edad mediana son parte de la evolución misma en la biología humana por lo que estos no justifican en sí mismo una perdida brusca y significativa de la actividad sexual, más bien si implican la readaptación y nuevos proyectos en el estilo de vida de los que necesitará la mujer, pues ninguna dificultad impide el placer pleno, dado que la respuesta sexual está afectada más por factores afectivos y cognitivos: fantasías, valoración de la relación, grado de intimidad, pasión sexual, entre otras (Argote, Mejía, Vásquez \& Villaquirán de González, 2009).

En los temas de Antropología del ciclo vital, se refiere que en la edad mediana se produce una autoevaluación donde la mujer valora hasta qué punto se ha acercado al cumplimiento de sus metas, es así como la autorrealización dependerá de la biografía de vida, de la ruta que la persona haya seguido en su vida hacia la satisfacción de sus necesidades, a la adaptación auto limitativa, a la expansión creadora y el mantenimiento del medio interno.

\section{REFERENCIAS}

Alfonso Rodríguez, A. C. (2010). Sexualidad. Salud sexual y determinantes sociales de la salud: notas para el debate. Revista Sexología y Sociedad, 16(42). Recuperado de http://revsexologiaysociedad.sld.cu/index.php/sexologiaysociedad/article/view/399/44 $\underline{11}$

Argote, L., Mejía, M., Vásquez, M. y Villaquirán de González, M. (2009). Climaterio y menopausia en mujeres afrodescendientes: una aproximación al cuidado desde su cultura. Aquichan, 8(1). Recuperado de

https://aquichan.unisabana.edu.co/index.php/aquichan/article/view/122/245 
Artiles, L. (2007). Ambiente, persona, sociedad, y cultura: Integralidad en el proceso de atención a la mujer de edad mediana. En: L. Artiles, D. A. Navarro \& B. R. Manzano Climaterio y menopausia: un enfoque desde lo social (pp. 58-65). La Habana: Científico-Técnica.

Así cambia el sexo según la edad. (2016 septiembre 20). Información. Recuperado de https://www.diarioinformacion.com/vida-y-estilo/salud/2016/05/25/varia-sexualidadedad/1765710.html

Bajo Arenas, J. M., Lailla Vicens, J. M. y Xercavins Montosa, J. (2009). Fundamentos de Ginecología. España: Sociedad Española de Ginecología y Obstetricia.

Casañas Velástegui, N. E. (2009). Psicología del docente. Recuperado de http://files.sld.cu/bmn/files/2018/04/PSICOLOGIA-DEL-DOCENTE.pdf

Couto Núñez, D. y Nápoles Méndez, D. (2014). Aspectos sociopsicológicos del climaterio y la menopausia. MEDISAN, 18(10), 1409-1418. Recuperado de http://scielo.sld.cu/scielo.php?script=sci arttext\&pid=S1029-30192014001000011

Davis, S. R. \& Tran, J. (2001). Testosterone influences libido and well being in women. Trends in Endocrinology \& Metabolism, 12(1), 33-37.

Espitia De La Hoz, F. J. y Orozco-Gallego, H. (2018). Fisiopatologia del trastorno del deseo sexual en el climaterio. Revista Médica de Risaralda, 24(1), 58-60. Recuperado de http://www.scielo.org.co/pdf/rmri/v24n1/v24n1a10.pdf

Federación Latinoamericana de Sociedades de Obstetricia y Ginecología (FLASOG). (2016). Climaterio y Menopausia. Ciudad México: Nieto. Recuperado de http://www.flasog.org/pt/static/libros/Libro-Climaterio-y-Menopausia-FLASOG.pdf

González Cárdenas, L. T., Bayarre Vera, H. D. y Hernández Melendez, E. (2019) Factores de riesgo de baja calidad de vida en mujeres con climaterio del Municipio Plaza de la Revolución de la Habana, Cuba. Archivos en Medicina Familiar, 21(1), 510. Recuperado de https://www.medigraphic.com/pdfs/medfam/amf-2019/amf191b.pdf

Hernández Sánchez, J. y Forero Bulla, C. M. (2011). Concepciones y percepciones sobre salud, calidad de vida, actividad física en una comunidad académica de salud. Revista Cubana de Enfermería, 27(2), 159-170. Recuperado de http://scielo.sld.cu/scielo.php?script=sci arttext\&pid=S0864-03192011000200008

Manzano Ovies, B. R. (2014). Climaterio. En: O. Rigor Ricardo y S. Santiesteban Alba, S. Obstetricia y Ginecología (pp. 223-231). La Habana: Ciencias Médicas.

McCabe, M. P., Sharlip, I. D., Lewis, R., Atalla, E., Balon, R., Fisher, A.D. ... Segraves, $R_{-}$T. (2016). Incidence and prevalence of sexual dysfunction in women and men: $A$ 
Consensus Statement from Fourth International Consultation on Sexual Medicine 2015. The Journal of Sexual Medicine; 13(2), 144-152.

Navarro Despaigne, D. y Fontaine Semanat, Y. (2001). Síndrome climatérico: su repercusión social en mujeres de edad mediana. Revista Cubana de Medicina General Integral, 17(2), 169-176. Recuperado de http://scielo.sld.cu/scielo.php?script=sci arttext\&pid=S0864-21252001000200010

Navarro Despaigne, D. A. (2001). Moduladores selectivos del receptor estrogénico: Su utilidad en la mujer posmenopáusica. Revista Cubana de Endocrinología, 12(2) Recuperado de http://scielo.sld.cu/scielo.php?script=sci arttext\&pid=S1561$\underline{29532001000200008}$

Navarro Despaigne, D. A., Méndez Gómez, N. y Duany Navarro, A. (2017). Menopausia y función sexual. En: B. Torres Rodríguez \& A.C. Alfonso Rodríguez (Edit.). Salud, malestares y problemas sexuales, textos y contextos. Diabetes mellitus y salud sexual. V.5. La Habana: CENESEX.

Nelson, H. D. (2008). Menopause. The Lancet. 371(9614), 760-770.

Orama Díaz, J. (2000). Lecturas de filosofía salud y sociedad. La Habana: ECIMED.

Organización Mundial de la Salud (OMS) (2019). Temas de Salud. Salud Sexual. Recuperado de https://www.who.int/topics/sexual health/es/

Oviedo Gilberto, L. (2004) La definición del concepto de percepción en psicología con base en la teoría Gestalt. Revista de Estudios Sociales, 18, 89-96. Recuperado de http://www.scielo.org.co/pdf/res/n18/n18a10.pdf

Sarduy Nápoles, M.R. \& Lugones Botell, M. (Eds). (2006). II Consenso cubano sobre climaterio y menopausia. Taller Nacional de Revisión y Actualización, 14-15 diciembre 2006; La Habana, Cuba Recuperado de http://www.sld.cu/galerias/pdf/sitios/ginecobs/consenso2006seccclimymenop.pdf

Sarmiento Leiva, M. (2000). Caracterización de la severidad del síndrome climatérico [tesis]. Santa Cruz del Norte: Facultad de Salud Pública.

Stalina Santisteban, A. (2011). Atención integral a las mujeres de edad mediana. Revista Cubana de Obstetricia y Ginecología, 37(2), 251-270. Recuperado de http://scielo.sld.cu/scielo.php?script=sci arttext\&pid=S0138-600X2011000200015 


\section{AUTORAS:}

Aime Napoles Betancourt

Especialista de 1er grado en Obstetricia y ginecología Hospital Nacional Enrique Cabrera, La Habana, Cuba.

aimenb@infomed.sld.cu

\section{Ada Caridad Alfonso Rodríguez}

Sociedad Cubana Multidisciplinaria para el Estudio de la Sexualualidad, La Habana Cuba. 